\title{
Frações físicas da matéria orgânica em Latossolo cultivado com cana-de-açúcar no Rio Grande do Sul
}

\author{
Roberta Jeske Kunde(1), Cláudia Liane Rodrigues de Lima ${ }^{(1)}$, \\ Sérgio Delmar dos Anjos Silva(2) e Clenio Nailto Pillon ${ }^{(2)}$
}

\begin{abstract}
(1)Universidade Federal de Pelotas, Faculdade de Agronomia Eliseu Maciel, Campus Universitário, s/no, Caixa Postal 354, CEP 96010900 Capão do Leão, RS, Brasil. E-mail: roberta_kunde@hotmail.com, clrlima@yahoo.com.br ${ }^{(2)}$ Embrapa Clima Temperado, Rodovia BR-392, Km 78, Monte Bonito, Caixa Postal 321, CEP 96010-971 Pelotas, RS, Brasil. E-mail: sergio.anjos@embrapa.br, clenio.pillon@embrapa.br
\end{abstract}

Resumo - O objetivo deste trabalho foi avaliar as frações físicas da matéria orgânica do solo (MOS) e o índice de manejo de carbono (IMC), após diferentes períodos de preparo do solo para a implantação de cana-deaçúcar. $\mathrm{O}$ estudo foi realizado em Latossolo Vermelho, de textura argilosa, depois de 1, 3 e 5 anos do preparo do solo para a implantação da cultura, com queima da palhada e colheita manual. Como referência, utilizou-se o solo de mata nativa adjacente à área de estudo. Amostras de solo foram coletadas nas camadas de $0,00-0,05$, 0,05-0,10 e 0,10-0,20 m, para quantificar o IMC, bem como o C orgânico total, o C particulado, o C associado a minerais e o $\mathrm{C}$ das frações leve livre, leve oclusa e pesada da MOS. O preparo do solo associado à queima da palhada na colheita diminuiu o aporte de matéria orgânica fresca ao solo e acelerou a decomposição do compartimento lábil da MOS, com reflexos negativos sobre os estoques das frações físicas do solo. As maiores proporções de $\mathrm{C}$ foram verificadas na fração associada a minerais. As frações densimétricas da MOS foram mais sensíveis na deteç̧ão de alterações dos estoques, labilidade, índice de labilidade e IMC, nos diferentes tempos de implantação da cultura. As áreas com cana-de-açúcar apresentam IMC inferior a 100, o que indica impacto negativo do cultivo sobre os estoques de matéria orgânica e sobre a qualidade física do solo.

Termos para indexação: Saccharum officinarum, fracionamento físico da matéria orgânica, índice de manejo de carbono, labilidade da matéria orgânica, qualidade física do solo.

\section{Physical fractions of organic matter in an Oxisol cultivated with sugarcane in the state of Rio Grande do Sul, Brazil}

\begin{abstract}
The objective of this work was to evaluate the physical fractions of soil organic matter (SOM) and the carbon management index (CMI) after different periods of soil tillage for sugarcane implantation. The study was carried out in a clayey Oxisol after 1, 3, and 5 years from soil tillage for crop plantation, with burning of the straw and manual cutting for harvest. As a reference, the soil of a native forest adjacent to the studied area was used. Soil samples were collected at the $0.00-0.05,0.05-0.10$, and $0.10-0.20-\mathrm{m}$ soil layers, in order to quantify the CMI, as well as total organic C, particulated C, C associated with minerals, and C in the free light, occluded light, and heavy fractions of SOM. Soil tillage associated with previous burning of straw reduced the input of fresh organic matter to the soil and accelerated the decomposition of the SOM labile compartment, negatively affecting the stocks of soil physical fractions. The highest $\mathrm{C}$ proportions were observed in the fraction associated with minerals. The SOM density fractions were more sensible to detect changes in the stocks, lability, and the CMI, in the different crop implantation times. The cultivated areas had CMI lower than 100, which indicates the negative impacts of cropping on the stocks of organic matter and on soil physical quality.
\end{abstract}

Index terms: Saccharum officinarum, physical fractionation of organic matter, carbon management index, lability of organic matter, soil physical quality.

\section{Introdução}

A cultura da cana-de-açúcar tem assumido grande importância com o aumento na demanda por energias renováveis, em substituição às de origem fóssil, na matriz energética nacional e internacional (Assunção et al., 2010; Rosseti et al., 2014). Entretanto, as técnicas de manejo da cultura utilizam vigoroso revolvimento do solo por ocasião do preparo e plantio. Esse manejo, associado aos diferentes sistemas de colheita, pode promover alterações significativas das propriedades físicas, químicas e biológicas do solo (Fagundes et al., 2014; Souza et al., 2014). 
A colheita da cultura da cana, atualmente, pode ser realizada com a cana queimada ou crua, de forma manual ou mecânica. Na colheita manual, a queima do canavial tem por objetivo facilitar as operações de corte. Entretanto, essa prática apresenta elevado impacto sobre o ambiente, pela menor adição de fitomassa ao solo, que afeta os estoques da matéria orgânica do solo (MOS) (Torres et al., 2014), e pelo aumento das emissões de $\mathrm{CO}_{2}$ para a atmosfera.

Por ser sensível a modificações impostas pelo uso e manejo do solo, a MOS é considerada um dos principais indicadores da sustentabilidade dos agroecossistemas, (Guimarães et al., 2012; Rossi et al., 2012). Além disso, ela é um componente básico da qualidade do solo, por seu papel essencial em diversos processos químicos, físicos e biológicos do solo (Mielniczuk, 2008). O estudo das frações físicas da MOS permite melhor avaliação dessa qualidade, pois elas exibem diferentes taxas de degradação bioquímica e microbiana e apresentam diferentes acessibilidade aos microrganismos e interações com a fase mineral do solo (Tan et al., 2007). Ou seja, por estar localizada em diferentes compartimentos, a MOS apresenta diferentes tempos de ciclagem e formas de proteção, e é constituída basicamente de frações lábeis e estáveis (Rangel \& Silva, 2007).

Técnicas de fracionamento físico da MOS têm possibilitado a identificação do acúmulo de C em compartimentos do solo (Santos et al., 2012; Conceição et al., 2014; Signor et al., 2014). No curto prazo, alterações da proporção das frações lábeis da MOS - como a do C orgânico particulado (COp), C da fração leve livre (FLL) e $\mathrm{C}$ da fração leve oclusa (FLO) - podem fornecer informações importantes sobre a sustentabilidade ambiental e a qualidade do solo, e possibilitar correções das estratégias de uso e manejo do solo (Santos et al., 2011).

Por meio do fracionamento granulométrico, é possível calcular o IMC que integra as variações nas diferentes frações da MOS (Nicoloso et al., 2008). Proposto por Blair et al. (1995), o IMC é uma medida relativa dos impactos do manejo do solo, quando este é comparado a uma situação original, como o solo sob floresta ou pastagem natural. O cálculo do IMC considera a labilidade da MOS e une características quantitativas e qualitativas para avaliar o desempenho de um determinado sistema de manejo. Valores de
IMC inferiores a 100 indicam práticas prejudicais à manutenção da MOS e da qualidade do solo.

Estudos que identifiquem as alterações da qualidade física do solo em sistemas de produção de cana, ao longo do tempo, ainda são escassos, principalmente no Estado do Rio Grande do Sul.

O objetivo deste trabalho foi avaliar as frações físicas da MOS e o IMC de um Latossolo Vermelho, cultivado com cana-de-açúcar por diferentes períodos, após o preparo do solo, em Salto do Jacuí, RS.

\section{Material e Métodos}

$\mathrm{O}$ estudo foi desenvolvido em áreas pertencentes à Destilaria Grandespe, no Município de Salto do Jacuí, RS. O solo das áreas experimentais foi classificado como Latossolo Vermelho distrófico típico (Santos et al., 2013), de textura argilosa (Tabela 1). Os tratamentos avaliados consistiram de avaliações a diferentes tempos da implantação, com preparo convencional, da cana-de-açúcar (Saccharum officinarum L.): C1, 1 ano; C3, 3 anos; e C5, 5 anos. Assim, o tratamento C1 foi avaliado após 1 ano do preparo convencional do solo, enquanto que o C5 foi avaliado após transcorridos 5 anos sem preparo do solo. Como referência, utilizouse uma área de mata nativa (MN) adjacente às áreas de estudo, com características e propriedades de solo e relevo similares.

As áreas experimentais localizam-se entre $28^{\circ} 59^{\prime} \mathrm{S}$ e $53^{\circ} 14^{\prime} \mathrm{W}$, com altitudes entre 349 e $369 \mathrm{~m}$. O clima da região, de acordo com a classificação climática de Köppen-Geiger, é subtropical úmido do tipo $\mathrm{Cfa}$, em que: $\mathrm{C}$ refere-se a clima temperado quente, com temperatura média do mês mais frio entre 3 e $18^{\circ} \mathrm{C}$; $\mathrm{f}$, ao fato de que em nenhum mês a precipitação é inferior a $60 \mathrm{~mm}$; e a, à temperatura do mês mais quente superior a $22^{\circ} \mathrm{C}$.

As áreas experimentais eram cultivadas com cana-de-açúcar desde 1988. O preparo convencional do solo consistiu em subsolagem a $0,50 \mathrm{~m}$, duas gradagens pesadas e abertura de sulcos com $0,25 \mathrm{~m}$ de profundidade. A adubação utilizada foi de $500 \mathrm{~kg} \mathrm{ha}^{-1}$ de N-P $\mathrm{O}_{5}-\mathrm{K}_{2} \mathrm{O}$ 5-25-25, conforme Araújo et al. (2013), em que $350 \mathrm{~kg}$ foram aplicados à linha de plantio, à profundidade de $0,30 \mathrm{~m}$, com uso de cultivador, e 150 $\mathrm{kg}$ foram aplicados a lanço. As cultivares plantadas foram SP 801842 e a RB 835089, e a colheita foi realizada manualmente, após a queima da palhada. 
Utilizou-se o delineamento experimental inteiramente casualizado, tendo-se coletado aleatoriamente, em dez pontos dentro de cada tratamento, amostras deformadas e indeformadas, à linha de plantio e a $0,00-0,05,0,05-0,10$, e 0,10 $0,20 \mathrm{~m}$ de profundidade. As amostras indeformadas foram coletadas com o auxílio de anéis volumétricos de $0,030 \times 0,048 \mathrm{~m}$, para a determinação da densidade do solo (Ds), conforme Donagema et al. (2011). As amostras deformadas foram coletadas com pá de corte e acondicionadas em sacos de plástico. Para a análise, elas foram espalhadas em bandejas e secas à sombra até atingir a umidade correspondente ao ponto de friabilidade, quando foram destorroadas manualmente, de forma suave, para não provocar compactação ou ruptura dos agregados.

Após a secagem, as amostras foram peneiradas em malha de 2,00 mm, para a determinação do C orgânico total (COT) e para o fracionamento granulométrico e densimétrico da MOS. Para a determinação do COT, as amostras de solo foram maceradas em gral de ágata, para a quantificação dos teores de $\mathrm{C}$ orgânico por oxidação a seco, em analisador elementar CHNS. Os resultados foram expressos quanto ao estoque $\left(\mathrm{Mg} \mathrm{ha}^{-1}\right)$, por meio de correção da massa equivalente,

Tabela 1. Teores de areia, silte e argila de Latossolo Vermelho, cultivado com cana-de-açúcar (Saccharum officinarum) após diferentes tempos de implantação da cultura, e sob mata nativa.

\begin{tabular}{|c|c|c|c|}
\hline \multirow[t]{2}{*}{ Tratamento $^{(1)}$} & Areia & Silte & Argila \\
\hline & \multicolumn{3}{|c|}{ - } \\
\hline & \multicolumn{3}{|c|}{ Camada $0,00-0,05 \mathrm{~m}$} \\
\hline $\mathrm{C} 1$ & 318,3 & 228,4 & 453,3 \\
\hline $\mathrm{C} 3$ & 239,0 & 254,6 & 506,4 \\
\hline $\mathrm{C} 5$ & 356,6 & 179,0 & 464,4 \\
\hline \multirow[t]{2}{*}{$\mathrm{MN}$} & 285,8 & 235,9 & 478,3 \\
\hline & \multicolumn{3}{|c|}{ Camada $0,05-0,10 \mathrm{~m}$} \\
\hline $\mathrm{C} 1$ & 310,7 & 228,9 & 460,4 \\
\hline $\mathrm{C} 3$ & 234,6 & 272,2 & 494,8 \\
\hline $\mathrm{C} 5$ & 310,6 & 211,4 & 472,6 \\
\hline \multirow[t]{2}{*}{$\mathrm{MN}$} & 265,6 & 267,8 & 466,5 \\
\hline & \multicolumn{3}{|c|}{ Camada $0,10-0,20 \mathrm{~m}$} \\
\hline $\mathrm{C} 1$ & 321,9 & 188,8 & 490,1 \\
\hline $\mathrm{C} 3$ & 221,6 & 266,0 & 512,4 \\
\hline $\mathrm{C} 5$ & 318,0 & 208,3 & 473,7 \\
\hline $\mathrm{MN}$ & 258,9 & 269,6 & 471,6 \\
\hline
\end{tabular}

tendo-se levado em conta as diferenças entre as massas de solo em cada camada, conforme Sisti et al. (2004).

O fracionamento granulométrico foi realizado conforme Cambardella \& Elliott (1992), tendo-se adicionado $60 \mathrm{~mL}$ de solução de hexametafosfato de sódio $\left(5 \mathrm{~g} \mathrm{~L}^{-1}\right)$ a $20 \mathrm{~g}$ de solo. As amostras foram homogeneizadas por 16 horas em agitador horizontal. Após a homogeneização, realizou-se o peneiramento úmido, em peneira de malha de $53 \mu \mathrm{m}$. O material retido na peneira consistiu de $\mathrm{C}$ orgânico particulado (COp), associado à fração areia, enquanto o material restante consistiu de $\mathrm{C}$ orgânico associado à fase mineral do solo (COam) correspondente às frações silte e argila. $\mathrm{O}$ material retido na peneira, após lavagem, foi transferido para placas de Petri e seco em estufa a $50^{\circ} \mathrm{C}$ por 24 horas. Após a secagem, o material foi submetido aos mesmos procedimentos já descritos quanto à determinação do COT. Os teores de COam foram determinados pela diferença entre o teor de COT e o de COp.

O fracionamento densimétrico foi realizado conforme Conceição et al. (2008), tendo-se adicionado $80 \mathrm{~mL}$ de solução de politungstato de sódio (PTS), com $2,0 \mathrm{~g} \mathrm{~cm}^{-3}$, a $10 \mathrm{~g}$ de solo, em tubo de centrífuga com $100 \mathrm{~mL}$. O tubo foi fechado com rolha $\mathrm{e}$ invertido lenta e manualmente por cinco vezes, para liberação da FLL, sem o rompimento dos agregados. A suspensão foi centrifugada a $2.000 \mathrm{~g}$, por $60 \mathrm{~min}$, e o sobrenadante com a FLL foi filtrado, a vácuo, em filtro Whatman GF/C previamente quantificado quanto à massa. Para retirar o excesso de sal (PTS), o filtro com a FLL foi lavado com água destilada e seco a $60^{\circ} \mathrm{C}$, por 24 horas, para que, então, se quantificasse a massa de filtro + FLL. Para separar a FLO, a solução de PTS foi retornada ao tubo que continha o pellet, que foi suspenso novamente e submetido à dispersão com ultrassom, mediante energia de $408 \mathrm{~J} \mathrm{~mL}^{-1}$, nas amostras das camadas $0,00-0,05 \mathrm{~m}$ e $0,05-0,10 \mathrm{~m}$, e de $299 \mathrm{~J} \mathrm{~mL}^{-1}$, nas da camada $0,10-0,20 \mathrm{~m}$. Estes níveis de energia são usados para obtenção da máxima dispersão das amostras em partículas primárias (Inda Junior et al., 2007). Após a dispersão, a suspensão foi novamente centrifugada ( $2.000 \mathrm{~g}$ por $60 \mathrm{~min}$ ), e a FLO foi obtida por filtragem, da mesma forma que a FLL. No presente estudo, a matéria orgânica particulada residual não foi quantificada, somente a fração pesada (FP). O carbono da FP foi obtido por diferença entre o COT e o C da FLL + C da FLO. 
Os teores de C presentes na FLL e na FLO foram quantificados por oxidação a seco, em analisador elementar CHNS, e os resultados foram expressos, quanto ao estoque $\left(\mathrm{Mg} \mathrm{ha}^{-1}\right)$, pela correção da massa equivalente, tendo-se levado em conta as diferenças entre as massas de solo em cada camada (Sisti et al., 2004).

A partir dos resultados dos estoques de $\mathrm{C}$, obtidos do fracionamento granulométrico e do fracionamento densimétrico, calculou-se o IMC de acordo Blair et al. (1995), com adaptações de Diekow et al. (2005), tendose considerado COp e FLL + FLO como representantes da fração lábil do COT, e Coam e FP como o representantes da fração não lábil. No presente estudo, considerou-se o IMC do solo da MN igual a $100 \%$. Para a obtenção do IMC, necessita-se do índice de estoque de C (IEC), que é calculado a partir da relação entre os estoques de $\mathrm{C}$ da área cultivada em relação ao estoque de $\mathrm{C}$ da área de referência. A labilidade da MOS foi determinada pela relação entre os estoques de COp e Coam, para o fracionamento granulométrico, e pela relação entre os estoques de FLL+FLO e FP, para o fracionamento densimétrico. $\mathrm{O}$ índice de labilidade (IL) foi calculado pela relação entre a labilidade de cada área com a área de referência. O IMC de cada área foi obtido por IMC = IEC x IL x 100.

Os resultados foram submetidos à análise de variância, e as médias foram comparadas pelo teste Tukey, a $5 \%$ de probabilidade.

\section{Resultados e Discussão}

Nas camadas $0,00-0,05$ e $0,05-0,10 \mathrm{~m}$, os maiores estoques de COT foram verificados na MN (Tabela 2), em razão da não utilização agrícola (menores taxas de decomposição) e das maiores taxas de adição de C ao solo (Amado et al., 2006; Pacheco \& Cantalice, 2011). De acordo com Góes et al. (2005), o intenso revolvimento do solo para a implantação da canade-açúcar aumenta a exposição da matéria orgânica fisicamente protegida à ação microbiana, o que acelera a oxidação e a decomposição da MOS, que diminui seu teor ao longo dos ciclos de cultivo.

Os menores estoques de COT, na camada 0,10 $0,20 \mathrm{~m}$, foram verificados na área com três anos (C3) de implantação (Tabela 2), o que está possivelmente associado ao efeito do preparo convencional do solo, que, para cana, atinge principalmente essa camada.
Independentemente da camada, os maiores valores de COp foram observados na MN (Tabela 2). Nas áreas cultivadas, não se observaram diferenças significativas quanto a esta variável, na camada $0,00-0,05 \mathrm{~m}$. Porém, nas camadas $0,05-0,10$ e $0,10-0,20 \mathrm{~m}$, os menores valores de COp foram observados em C3. Esse resultado pode estar associado ao menor aporte de biomassa vegetal, em razão da queima da palhada. $\mathrm{Na} \mathrm{MN}$, o aporte constante de resíduos vegetais sobre a superfície do solo, aliado à não perturbação do sistema, contribuiu para os maiores valores de COp (Müller et al., 2012; Rosseti et al., 2014). Segundo Rossi et al. (2012), sistemas com maior aporte de biomassa acumulam $C$ preferencialmente na fração particulada, que representa uma variável mais sensível às alterações de manejo em curto prazo do que o COT. Silva et al. (2006), ao avaliar a matéria orgânica e as propriedades físicas de um Argissolo em sistemas de manejo com cana-de-açúcar, constataram que o cultivo reduziu o teor de COT e de COp, em comparação à MN. Galdos

Tabela 2. Estoques de $\mathrm{C}$ orgânico total (COT), C orgânico particulado (COp), C orgânico associado a minerais (COam), $\mathrm{C}$ da fração leve livre (FLL), $\mathrm{C}$ da fração leve oclusa (FLO) e C da fração pesada (FP), em Latossolo Vermelho cultivado com cana-de-açúcar (Saccharum officinarum), após diferentes tempos da implantação da cultura, e sob mata nativa ${ }^{(1)}$.

\begin{tabular}{|c|c|c|c|c|c|c|}
\hline Tratamento $^{(2)}$ & COT & CFG & COam & FLL & FLO & FP \\
\hline & \multicolumn{6}{|c|}{ Camada $0,00-0,05 \mathrm{~m}$} \\
\hline $\mathrm{C} 1$ & $11,44 b$ & $2,11 \mathrm{~b}$ & $9,33^{\mathrm{ns}}$ & $0,76 \mathrm{~b}$ & $1,52 b$ & $9,16 \mathrm{~b}$ \\
\hline $\mathrm{C} 3$ & $11,25 b$ & $1,76 b$ & 9,50 & $0,64 b$ & $1,57 b$ & $9,04 \mathrm{~b}$ \\
\hline $\mathrm{C} 5$ & $11,00 \mathrm{~b}$ & $2,16 \mathrm{~b}$ & 8,83 & $0,68 \mathrm{~b}$ & $1,83 \mathrm{~b}$ & $8,50 \mathrm{~b}$ \\
\hline $\mathrm{MN}$ & $17,86 \mathrm{a}$ & $7,60 \mathrm{a}$ & 10,26 & $2,88 \mathrm{a}$ & $2,80 \mathrm{a}$ & $12,18 \mathrm{a}$ \\
\hline \multirow[t]{2}{*}{ CV $(\%)$} & 15,17 & 19,00 & 18,00 & 15,00 & 11,87 & 20,00 \\
\hline & \multicolumn{6}{|c|}{ Camada $0,05-0,10 \mathrm{~m}$} \\
\hline $\mathrm{C} 1$ & $11,09 \mathrm{~b}$ & $1,77 \mathrm{~b}$ & $9,31 \mathrm{~b}$ & $0,37 \mathrm{c}$ & $1,43 \mathrm{~d}$ & $9,27^{\text {ns }}$ \\
\hline $\mathrm{C} 3$ & $11,22 b$ & $1,21 \mathrm{c}$ & $10,01 \mathrm{ab}$ & $0,28 \mathrm{c}$ & $1,68 \mathrm{c}$ & 9,25 \\
\hline $\mathrm{C} 5$ & $11,69 \mathrm{~b}$ & $1,91 \mathrm{~b}$ & $9,78 \mathrm{ab}$ & $0,78 \mathrm{~b}$ & $1,96 \mathrm{~b}$ & 8,96 \\
\hline MN & $14,31 \mathrm{a}$ & $3,42 \mathrm{a}$ & $10,89 \mathrm{a}$ & $1,39 \mathrm{a}$ & $2,33 \mathrm{a}$ & 10,59 \\
\hline \multirow[t]{2}{*}{ CV (\%) } & 13,00 & 15,00 & 13,07 & 10,88 & 5,04 & 16,00 \\
\hline & \multicolumn{6}{|c|}{ Camada $0,10-0,20 \mathrm{~m}$} \\
\hline $\mathrm{C} 1$ & $23,32 \mathrm{a}$ & $3,44 b$ & $19,88^{\text {ns }}$ & $0,85 \mathrm{~b}$ & $3,32 b$ & $19,15^{\text {ns }}$ \\
\hline $\mathrm{C} 3$ & $20,77 b$ & $1,86 \mathrm{c}$ & 18,91 & $0,39 \mathrm{c}$ & $2,43 \mathrm{c}$ & 17,96 \\
\hline $\mathrm{C} 5$ & $23,34 \mathrm{a}$ & $3,32 b$ & 20,02 & $0,94 \mathrm{~b}$ & $3,52 b$ & 17,88 \\
\hline MN & $22,85 \mathrm{a}$ & $3,94 a$ & 18,90 & $1,54 \mathrm{a}$ & $4,68 \mathrm{a}$ & 16,69 \\
\hline $\mathrm{CV}(\%)$ & 7,59 & 9,55 & 8,96 & 8,28 & 6,39 & 9,21 \\
\hline
\end{tabular}

Pesq. agropec. bras., Brasília, v.51, n.9, p.1520-1528, set. 2016 DOI: 10.1590/S0100-204X2016000900051 
et al. (2009) também obtiveram maiores estoques de COp na área com MN, em todas as camadas, em comparação a áreas com cana-de-açúcar com ou sem queima da palhada. Os autores também relataram que os decréscimos dos estoques de COp acentuaram-se com o tempo de cultivo da cana-de-açúcar com queima da palhada. Silva et al. (2007), observaram redução de $20 \%$ dos estoques de COp, após 25 anos de cultivo com queima da palhada, em Latossolo Vermelho.

O COp, em geral, apresenta-se em menor proporção do que o COam, com valores em torno de 3 a $20 \%$ do COT no solo. No entanto, os estoques desta fração podem aumentar em sistemas com menor revolvimento do solo e em condições climáticas menos favoráveis à decomposição da MOS (Silva \& Mendonça, 2007). De fato, na camada superficial $(0,00-0,05 \mathrm{~m})$ da área com MN, o COp representou 43\% do COT (Tabela 3 ). As áreas cultivadas apresentaram proporção de COp de 9 a 20\% do COT. Galdos et al. (2009) observaram proporção média de $23,8 \%$. De acordo com os autores, diferenças nessa proporção podem estar associadas ao tipo de solo, às condições climáticas e à qualidade $\mathrm{e}$ magnitude do aporte de resíduos culturais, bem como

Tabela 3. Proporções de $\mathrm{C}$ orgânico particulado (COp), associada a minerais (COam), leve livre (FLL), leve oclusa (FLO) e pesada (FP), em relação ao C orgânico total (COT) em Latossolo Vermelho, cultivado com cana-de-açúcar (Saccharum officinarum), após diferentes tempos do preparo para implantação da cultura, e sob mata nativa.

\begin{tabular}{|c|c|c|c|c|c|}
\hline Tratamento $^{(1)}$ & $\mathrm{CFG/COT}$ & $\mathrm{COam} / \mathrm{COT}$ & FLL/COT & $\mathrm{FLO} / \mathrm{COT}$ & $\mathrm{FP} / \mathrm{COT}$ \\
\hline & \multicolumn{5}{|c|}{ Camada $0,00-0,05 \mathrm{~m}$} \\
\hline $\mathrm{C} 1$ & 18 & 82 & 7 & 13 & 80 \\
\hline $\mathrm{C} 3$ & 16 & 84 & 6 & 14 & 80 \\
\hline $\mathrm{C} 5$ & 20 & 80 & 6 & 17 & 77 \\
\hline \multirow[t]{2}{*}{$\mathrm{MN}$} & 43 & 57 & 15 & 17 & 68 \\
\hline & \multicolumn{5}{|c|}{ Camada $0,05-0,10 \mathrm{~m}$} \\
\hline $\mathrm{C} 1$ & 16 & 84 & 3 & 13 & 84 \\
\hline $\mathrm{C} 3$ & 11 & 89 & 2 & 15 & 83 \\
\hline $\mathrm{C} 5$ & 16 & 84 & 7 & 17 & 76 \\
\hline \multirow[t]{2}{*}{$\mathrm{MN}$} & 24 & 76 & 10 & 16 & 74 \\
\hline & \multicolumn{5}{|c|}{ Camada $0,10-0,20 \mathrm{~m}$} \\
\hline $\mathrm{C} 1$ & 15 & 85 & 4 & 14 & 82 \\
\hline $\mathrm{C} 3$ & 9 & 91 & 2 & 12 & 86 \\
\hline $\mathrm{C} 5$ & 14 & 86 & 4 & 15 & 81 \\
\hline $\mathrm{MN}$ & 17 & 83 & 7 & 20 & 73 \\
\hline
\end{tabular}

${ }^{(1)}$ Tempo após preparo do solo para a implantação da cultura da cana-de-açúcar: C1, após um ano; C3, após 3 anos; C5, após 5 anos. MN: mata nativa. à intensidade de revolvimento do solo. No presente trabalho, a proporção de COp em relação ao COT diminuiu em profundidade, independentemente do tempo de implantação da cultura.

O tempo de implantação não causou diferenças significativas dos estoques de COam, a $0,00-0,05$ e $0,10-0,20 \mathrm{~m}$ (Tabela 2) de profundidade. Na camada 0,05-0,10 m, o solo com $\mathrm{MN}$ apresentou valores maiores dessa variável do que $\mathrm{C} 1$, mas não diferiu estatisticamente de C3 nem de C5. Normalmente, o COam mostra-se menos sensível ao manejo do solo, principalmente no curto prazo (Bayer et al., 2004). Entretanto, o resultado quanto ao COam evidenciou que a proximidade do preparo do solo, como em $\mathrm{C} 1$, estimulou perdas de $\mathrm{C}$, uma vez que houve curto período para a reestabilização da matéria orgânica nessa fração.

O solo analisado apresentava cerca de $50 \%$ de argila, indício de considerável potencial de associação da MO a minerais do solo. Solos com maior conteúdo de argila têm maior poder de conservação e estabilização da MOS (Carmo et al., 2012). Além disso, o solo avaliado apresentava elevados teores de óxidos e hidróxidos de $\mathrm{Fe}$ e $\mathrm{Al}$, o que contribuiu ainda mais para a estabilização da MOS. A maior parte do COT esteve ligada aos minerais, com valores de COam entre 57 e 91\% (Tabela 3).

Com relação ao fracionamento densimétrico da MOS, observou-se que os maiores valores da FLL e FLO foram observados na $\mathrm{MN}$, em todas as camadas (Tabela 2). Os menores valores de FLL ocorreram nas áreas $\mathrm{C} 1$ e $\mathrm{C} 3$, a $0,05-0,10 \mathrm{~m}$ de profundidade, , enquanto a FLO foi menor em C1. Estes resultados também estão associados à maior proximidade do preparo do solo, que expõe a matéria orgânica lábil (FLL +FLO) à decomposição microbiana.

Os menores valores de FLL e FLO foram observados na C3, na camada $0,10-0,20 \mathrm{~m}$ (Tabela 2). Assim como o observado nas frações físicas granulométricas, os menores estoques destas frações em $\mathrm{C} 3$ podem estar relacionados ao fato de o manejo convencional atingir principalmente a camada $0,10-0,20 \mathrm{~m}$.

Segundo Six et al. (2002), a FLL é composta por resíduos parcialmente decompostos e é fortemente influenciada pela quantidade e qualidade de resíduos depositados no solo. Uma vez que o mecanismo de proteção da FLL no solo consiste apenas de sua recalcitrância molecular, o estoque dessa fração depende da adição de resíduos e da velocidade de 
decomposição, e seu tempo de permanência no sistema é curto, pela facilidade de degradação.

Os maiores valores de FP foram observados na $\mathrm{MN}$, a $0,00-0,05 \mathrm{~m}$ de profundidade (Tabela 2). Nas demais camadas, não se verificaram diferenças significativas entre os tratamentos. Como a FP apresenta ciclagem mais lenta, em razão da estabilização em superfícies minerais do solo e da proteção física no interior de agregados estáveis, seria necessário um período maior do que o avaliado neste trabalho, para que o preparo do solo pudesse ter efeito sobre os teores de $\mathrm{C}$ desta fração (Bayer et al., 2004).

As proporções de COT decresceram na ordem FP $>$ FLL $>$ FLO, na $\mathrm{MN}$, na camada superficial $(0,00-0,05 \mathrm{~m})$ (Tabela 3). Portanto, o mecanismo de recalcitrância molecular e de interação do material orgânico com os minerais do solo foram mais relevantes do que a estabilização decorrente da oclusão em agregados (FLO, 17\%). No entanto, nas demais camadas e nos solos cultivados, as proporções de COT decresceram na ordem FP $>$ FLO $>$ FLL. Assim, as maiores proporções de COT estiveram sempre na $\mathrm{FP}$, o que é justificável, já que nela atuam os três mecanismos de proteção: recalcitrância molecular, proteção física e proteção química ou coloidal (Roscoe \& Machado, 2002).
As proporções de FLL em relação ao COT variaram de 2 à 15\% (Tabela 3). Conceição et al. (2008) relataram que o mecanismo de recalcitrância molecular da MOS, presente na FLL, tem importância limitada diante da estabilidade decorrente da proteção física em agregados (FLO) e da interação com os minerais do solo (FP).

As menores proporções de FP foram verificadas na MN (Tabela 3), independentemente da camada avaliada; isso se explica pelo acúmulo da MOS em frações mais lábeis (FLL e FLO), em sistemas não antropizados.

Os maiores IEC, a 0,00 a $0,10 \mathrm{~m}$ de profundidade, foram obtidos na MN (Tabela 4). Na camada $0,10-0,20$ $\mathrm{m}$, os menores valores de IEC foram obtidos na área $\mathrm{C} 1$, e os resultados para a variável seguiram a tendência dos obtidos para o COT.

Ao aportarem diferentes quantidades de $\mathrm{C}$ ao solo, os sistemas de manejo alteram a labilidade (L) da MOS, ou seja, alteram a proporção de matéria orgânica lábil em relação à não lábil. Em ambos os métodos de fracionamento avaliados, os valores de $\mathrm{L}$ e IL não diferiram entre as áreas cultivadas, na camada 0,00-0,05 m, mas foram menores do que os obtidos na área de referência (Tabela 4). Este resultado pode ser justificado pelo menor aporte de resíduos nas áreas cultivadas, principalmente em razão da queima da

Tabela 4. Índice de estoque de C (IEC), labilidade da matéria orgânica (L), índice de labilidade (IL) e índice de manejo de C (IMC), em Latossolo Vermelho cultivado com cana-de-açúcar (Saccharum officinarum) após diferentes tempos da implantação da cultura, e sob mata nativa ${ }^{(1)}$.

\begin{tabular}{|c|c|c|c|c|c|c|c|}
\hline \multirow[t]{2}{*}{ Tratamento $^{(2)}$} & \multirow[t]{2}{*}{ IEC } & \multicolumn{3}{|c|}{ Granulométrico } & \multicolumn{3}{|c|}{ Densimétrico } \\
\hline & & $\mathrm{L}$ & IL & IMC & $\mathrm{L}$ & IL & IMC \\
\hline & \multicolumn{7}{|c|}{ Camada $0,00-0,05 \mathrm{~m}$} \\
\hline $\mathrm{C} 1$ & $0,64 b$ & $0,23 b$ & $0,43 b$ & $27,50 \mathrm{~b}$ & $0,92 b$ & $0,29 b$ & $18,87 \mathrm{~b}$ \\
\hline $\mathrm{C} 3$ & $0,63 b$ & $0,18 b$ & $0,35 b$ & $22,31 b$ & $0,82 b$ & $0,28 b$ & $17,43 b$ \\
\hline $\mathrm{C} 5$ & $0,62 \mathrm{~b}$ & $0,25 \mathrm{~b}$ & $0,47 \mathrm{~b}$ & $29,34 \mathrm{~b}$ & $0,88 \mathrm{~b}$ & $0,26 \mathrm{c}$ & $16,41 \mathrm{c}$ \\
\hline \multirow[t]{2}{*}{$\mathrm{MN}$} & $1,00 \mathrm{a}$ & $0,53 \mathrm{a}$ & $1,00 \mathrm{a}$ & $100,00 \mathrm{a}$ & $3,14 \mathrm{a}$ & $1,00 \mathrm{a}$ & $100,00 \mathrm{a}$ \\
\hline & \multicolumn{7}{|c|}{ Camada $0,05-0,10 \mathrm{~m}$} \\
\hline $\mathrm{C} 1$ & $0,77 \mathrm{~b}$ & $0,19 b$ & $0,58 b$ & $45,44 b$ & $0,53 \mathrm{c}$ & $0,33 \mathrm{c}$ & $25,25 \mathrm{c}$ \\
\hline $\mathrm{C} 3$ & $0,79 b$ & $0,12 \mathrm{c}$ & $0,37 \mathrm{c}$ & $29,22 \mathrm{c}$ & $0,46 \mathrm{c}$ & $0,28 \mathrm{c}$ & $24,26 \mathrm{c}$ \\
\hline $\mathrm{C} 5$ & $0,82 b$ & $0,20 \mathrm{~b}$ & $0,60 \mathrm{~b}$ & $48,98 \mathrm{~b}$ & $0,79 b$ & $0,61 b$ & $50,11 \mathrm{~b}$ \\
\hline \multirow[t]{2}{*}{ MN } & $1,00 \mathrm{a}$ & $0,33 \mathrm{a}$ & $1,00 \mathrm{a}$ & $100,00 \mathrm{a}$ & $1,62 \mathrm{a}$ & $1,00 \mathrm{a}$ & $100,00 \mathrm{a}$ \\
\hline & \multicolumn{7}{|c|}{ Camada $0,10-0,20 \mathrm{~m}$} \\
\hline $\mathrm{C} 1$ & $1,02 \mathrm{a}$ & $0,17 \mathrm{~b}$ & $0,81 b$ & $83,27 \mathrm{~b}$ & $0,17 \mathrm{~b}$ & $0,56 \mathrm{c}$ & $57,05 b$ \\
\hline $\mathrm{C} 3$ & $0,90 \mathrm{~b}$ & $0,09 \mathrm{c}$ & $0,46 \mathrm{c}$ & $41,94 \mathrm{c}$ & $0,09 \mathrm{c}$ & $0,28 \mathrm{~d}$ & $26,14 \mathrm{c}$ \\
\hline $\mathrm{C} 5$ & $1,02 \mathrm{a}$ & $0,17 \mathrm{~b}$ & $0,77 \mathrm{~b}$ & $79,69 \mathrm{~b}$ & $0,17 \mathrm{~b}$ & $0,61 \mathrm{~b}$ & $63,10 \mathrm{~b}$ \\
\hline $\mathrm{MN}$ & $1,00 \mathrm{a}$ & $0,21 \mathrm{a}$ & $1,00 \mathrm{a}$ & $100,00 \mathrm{a}$ & $0,21 \mathrm{a}$ & $1,00 \mathrm{a}$ & $100,00 \mathrm{a}$ \\
\hline
\end{tabular}


palhada. Nas camadas mais profundas, os menores valores de L e IL foram encontrados em C1, de acordo com o fracionamento granulométrico. Este resultado está associado aos menores estoques de COp obtidos nessa área. Com o fracionamento densimétrico, os valores de L e IL seguiram a mesma tendência do granulométrico, na camada $0,10-0,20 \mathrm{~m}$. Entretanto, na camada $0,05-0,10 \mathrm{~m}$, ele foi mais sensível na identificação de diferenças entre os tratamentos, com os menores valores de L e IL obtidos em C1 e C3.

Em todas as áreas, a labilidade da MOS diminuiu com a profundidade, tendo chegado a valores inferiores a 0,1 (Tabela 4). Schiavo et al. (2011), ao avaliar o IMC e atributos químicos de um Latossolo, verificaram que a labilidade da MO diminuiu com o aumento da profundidade, independentemente da cobertura.

Os maiores valores de IMC foram obtidos na área com MN, com ambos os tipos de fracionamento. No granulométrico, às áreas cultivadas tiveram os menores valores de IMC nas camadas $0,05-0,10$ e $0,10-0,20 \mathrm{~m}$, especialmente na área $\mathrm{C} 1$ (Tabela 4). Entretanto, com o fracionamento densimétrico, que possibilitou maior sensibilidade do índice na distinção dos tratamentos, os menores valores na camada $0,00-0,05$ e $0,10-0,20 \mathrm{~m}$ foram obtidos em $\mathrm{C} 3$, e, na camada $0,05-0,10 \mathrm{~m}$, em $\mathrm{C} 1$ e C3.

Blair et al. (1995), em estudo com Argissolo (Ultisol) cultivado com cana queimada por 90 anos, em Marian, na Austrália, encontraram IMC igual a 34. Entretanto, quando a palhada da cana-de-açúcar foi mantida sem queima sobre o solo, os autores relataram aumento de $48 \%$ do IMC em um Latossolo (Oxisol), em comparação ao sistema de referência. De fato, Blair (2000) relatou maiores valores de IMC, em diferentes sistemas de manejo de cana-de-açúcar na Austrália, em áreas sem queima.

Em todas as camadas, independentemente do sistema de fracionamento, o IMC das áreas cultivadas foi inferior à 100 (Tabela 4), o que indica que o sistema de manejo utilizado, com queima da palhada na colheita, prejudicou a manutenção da matéria orgânica e da qualidade do solo.

\section{Conclusões}

1. O preparo do solo para a implantação da cana-deaçúcar, com emprego da queima da palhada na colheita, diminui o aporte de matéria orgânica fresca sobre o solo e acelera a decomposição do compartimento lábil da matéria orgânica do solo (MOS), com reflexos negativos sobre os estoques das frações físicas do solo.

2. Independentemente do tipo de fracionamento, as maiores proporções do $\mathrm{C}$ orgânico são verificadas na fração associada aos minerais do solo (C orgânico associado aos minerais $+\mathrm{C}$ da fração pesada).

3. As frações físicas da MOS obtidas por meio do fracionamento densimétrico apresentam maior sensibilidade em detectar alterações dos estoques, labilidade, índice de labilidade e índice de manejo de $\mathrm{C}$, nos diferentes tempos após o preparo do solo para implantação da cana-de-açúcar.

4. Todas as áreas cultivadas com cana-de-açúcar apresentam índice de manejo de C inferior a 100 , principalmente nas áreas com 1 e 3 anos após o preparo, o que indica impacto negativo do manejo do solo sobre a qualidade física dessas áreas.

\section{Agradecimentos}

Ao Conselho Nacional de Desenvolvimento Científico e Tecnológico (CNPq) e a Financiadora de Estudos e Projetos (Finep), pelo apoio financeiro.

\section{Referências}

AMADO, T.J.C.; BAYER, C.; CONCEIÇÃO, P.C.; SPAGNOLLO, E.; CAMPOS, B.H.C. de; VEIGA, M. da. Potential of carbon accumulation in no-till soils with intensive use and cover crops in southern Brazil. Journal of Enviromental Quality, v.35, p.15991607, 2006. DOI: 10.2134/jeq2005.0233.

ARAÚJO, K.L.; CANTERI, M.G.; GILIO, T.A.S.; NEUBAUER, R.A.; SANCHES, P.B.; SUMIDA, C.H.; GIGLIOTI, E.A. Resistência genotípica e monitoramento da favorabilidade para ocorrência da ferrugem alaranjada da cana-de-açúcar. Summa Phytopathologica, v.39, p.271-275, 2013. DOI: 10.1590/S010054052013000400007.

ASSUNÇÃO, A.; SANTOS, L. de C.; ROCHA, M.R. da; REIS, A.J. dos S.; TEIXEIRA, R.A.; LIMA, F.S. de O. Efeito de indutores de resistência sobre Meloidogyne incognita em cana-de-açúcar (Saccharum spp.). Revista Nematologia Brasileira, v.34, p.5662, 2010.

BAYER, C.; MARTIN-NETO, L.; MIELNICZUK, J.; PAVINATO, A. Armazenamento de carbono em frações lábeis da matéria orgânica de um Latossolo Vermelho sob plantio direto. Pesquisa Agropecuária Brasileira, v.39, p.677-683, 2004. DOI: 10.1590/ S0100-204X2004000700009.

BLAIR, G.J.; LEFROY, R.D.B.; LISLE, L. Soil carbon fractions based on their degree of oxidation, and the development of a carbon management index for agricultural systems. Australian 
Journal of Agricultural Research, v.46, p.1459-1466, 1995. DOI: 10.1071/AR9951459.

BLAIR, N. Impact of cultivation and sugar-cane green trash management on carbon fractions and aggregate stability for a Chromic Luvisol in Queensland, Australia. Soil and Tillage Research, v.55, p.183-191, 2000. DOI: 10.1016/S01671987(00)00113-6.

CAMBARDELLA, C.A.; ELLIOTT, E.T. Particulate soil organicmatter changes across a grassland cultivation sequence. Soil Science Society of America Journal, v.56, p.777-783, 1992. DOI: 10.2136/sssaj 1992.03615995005600030017x.

CARMO, F.F. do; FIGUEIREDO, C.C. de; RAMOS, M.L.G.; VIVALDI, L.J.; ARAÚJO, L.G. Frações granulométricas da matéria orgânica em Latossolo sob plantio direto com gramíneas. Bioscience Journal, v.28, p.420-431, 2012.

CONCEIÇÃO, P.C.; BAYER, C.; DIECKOW, J.; SANTOS, D.C. dos. Fracionamento físico da matéria orgânica e índice de manejo de carbono de um Argissolo submetido a sistemas conservacionistas de manejo. Ciência Rural, v.44, p.794-800, 2014. DOI: 10.1590/ S0103-84782014005000004.

CONCEIÇÃO, P.C.; BOENI, M.; DIECKOW, J.; BAYER, C.; MIELNICZUK, J. Fracionamento densimétrico com politungstato de sódio no estudo da proteção física da matéria orgânica em solos. Revista Brasileira de Ciência do Solo, v.32, p.541-549, 2008. DOI: 10.1590/S0100-06832008000200009.

DIEKOW, J.; MIELNICZUK, J.; KNICKER, H.; BAYER, C.; DICK, D.P.; KÖGEL-KNABER, I. Carbon and nitrogen stocks in physical fractions of a subtropical Acrisol as influenced by longterm no-till cropping systems and $\mathrm{N}$ fertilisation. Plant and Soil, v.268, p.319-328, 2005. DOI: 10.1007/s11104-004-0330-4.

DONAGEMA, G.K.; CAMPOS, D.V.B. de; CALDERANO, S.B.; TEIXEIRA, W.G.; VIANA, J.H.M. (Org.). Manual de métodos de análise de solo. 2.ed. rev. Rio de Janeiro: Embrapa Solos, 2011. 212p. (Embrapa Solos. Documentos, 132).

FAGUNDES, E.A.A.; SILVA, T.J.A. da; BONFIM-SILVA, E.M. Desenvolvimento inicial de variedades de cana-de-açúcar em Latossolo submetidas a níveis de compactação do solo. Revista Brasileira de Engenharia Agrícola e Ambiental, v.18, p.188193, 2014. DOI: 10.1590/S1415-43662014000200009.

GALDOS, M.V.; CERRI, C.C.; CERRI, C.E.P. Soil carbon stocks under burned and unburned sugarcane in Brazil. Geoderma, v.153, p.347-352, 2009. DOI: 10.1016/j.geoderma.2009.08.025.

GÓES, G.B. de; GREGGIO, T.C.; CENTURION, J.F.; BEUTLER, A.N.; ANDRIOLI, I. Efeito do cultivo da cana-de-açúcar na estabilidade de agregados e na condutividade hidráulica do solo. Irriga, v.10, p.116-122, 2005.

GUIMARÃES, D.V.; GONZAGA, M.I.S.; MELO NETO, J.O.; REIS, A.F.; LIMA, T.S.; SANTANA, I.L. Qualidade da matéria orgânica do solo e estoques de carbono e nitrogênio em fragmento de Mata Atlântica do Município de Neópolis, Sergipe. Scientia Plena, v.8, p.1-5, 2012.

INDA JUNIOR, A.V.; BAYER, C.; CONCEIÇÃO, P.C.; BOENI, M.; SALTON, J.C.; TONIN, A.T. Variáveis relacionadas à estabilidade de complexos organo-minerais em solos tropicais e subtropicais brasileiros. Ciência Rural, v.37, p.1301-1307, 2007. DOI: $10.1590 / \mathrm{S} 0103-84782007000500013$.

MIELNICZUK, J. Matéria orgânica e a sustentabilidade de sistemas agrícolas. In: SANTOS, G. de A; SILVA.L.S. da; CANELLAS, L.P.; CAMARGO, F. de O. (Ed.). Fundamentos da matéria orgânica do solo: ecossistemas tropicais e subtropicais. 2.ed. rev. e atual. Porto Alegre: Metrópole, 2008. p.1-5.

MÜLLER, C.B.; WEBER, O.L. dos S.; SCARAMUZZA, J.F. Oxidable fraction of organic carbon in an Argisol under different land use systems. Cerne, v.18, p.215-222, 2012. DOI: 10.1590/ S0104-77602012000200005.

NICOLOSO, R. da S. LOVATO, T.; AMADO, T.J.C.; BAYER, C.; LANZANOVA, M.E. Balanço do carbono orgânico no solo sob integração lavoura-pecuária no Sul do Brasil. Revista Brasileira de Ciência do Solo, v.32, p.2425-2433, 2008. DOI: 10.1590/ S0100-06832008000600020.

PACHECO, E.P.; CANTALICE, J.R.B. Análise de trilha no estudo dos efeitos de atributos físicos e da matéria orgânica sobre a compressibilidade e resistência à penetração de um Argissolo cultivado com cana-de-açúcar. Revista Brasileira de Ciência do Solo, v.35, p.417-428, 2011. DOI: 10.1590/S010006832011000200011.

RANGEL, O.J.P.; SILVA, C.A. Estoques de carbono e nitrogênio e frações orgânicas de latossolo submetido a diferentes sistemas de uso e manejo. Revista Brasileira de Ciência do Solo, v.31, p.1609-1623, 2007. DOI: 10.1590/S0100-06832007000600037.

ROSCOE, R.; MACHADO, P.L.O. de A. Fracionamento físico do solo em estudos da matéria orgânica. Dourados: Embrapa Agropecuária Oeste; Rio de Janeiro: Embrapa Solos, 2002. 86p.

ROSSETI, K. de V.; TEIXEIRA, D.D.B.; REIS, I.M.S.; CENTURION, J.F. Agregação de um Latossolo em função de diferentes ciclos de cultivo de cana-de-açúcar sob colheita mecanizada. Revista Agro@mbiente, v.8, p.10-17, 2014. DOI: 10.18227/1982-8470ragro.v8i1.1438.

ROSSI, C.Q.; PEREIRA, M.G.; GIÁCOMO, S.G.; BETTA, M.; POLIDORO, J.C. Frações lábeis da matéria orgânica em sistema de cultivo com palha de braquiária e sorgo. Revista Ciência Agronômica, v.43, p.38-46, 2012.

SANTOS, D.C. dos; LIMA, C.L.; KUNDE, R.J.; CARVALHO, J.S.; ABEIJON, L.M.; PILLON, C.N. Agregação e proteção física da matéria orgânica em Planossolo Háplico sob diferentes sistemas de manejo. Bioscience Journal, v.28, p.54-63, 2012.

SANTOS, D.C. dos; PILLON, C.N.; FLORES, C.A.; LIMA, C.L.R. de; CARDOSO, E.M.C.; PERREIRA, B.F.; MANGRICH, A.S. Agregação e frações físicas da matéria orgânica de um Argissolo Vermelho sob sistemas de uso no bioma Pampa. Revista Brasileira de Ciência do Solo, v.35, p.1735-1744, 2011. DOI: 10.1590/S0100-06832011000500028.

SANTOS, H.G. dos; JACOMINE, P.K.T.; ANJOS, L.H.C. dos; OLIVEIRA, V.A. de; LUMBRERAS, J.F.; COELHO, M.R.; ALMEIDA, J.A. de; CUNHA, T.J.F.; OLIVEIRA, J.B. de. Sistema brasileiro de classificação de solos. 3.ed. rev. e ampl. Brasília: Embrapa, 2013. 353p. 
SCHIAVO, J.A.; ROSSET, J.S.; PEREIRA, M.G.; SALTON, J.C. Índice de manejo de carbono e atributos químicos de Latossolo Vermelho sob diferentes sistemas de manejo. Pesquisa Agropecuária Brasileira, v.46, p.1332-1338, 2011. DOI: 10.1590/ S0100-204X2011001000029.

SIGNOR, D.; ZANI, C.F.; PALADINI, A.A.; DEON, M.D.I.; CERRI, C.E.P. Estoques de carbono e qualidade da matéria orgânica do solo em áreas cultivadas com cana-de-açúcar. Revista Brasileira de Ciência do Solo, v.38, p.1402-1410, 2014. DOI: 10.1590/S0100-06832014000500005.

SILVA, A.J.N. da; CABEDA, M.S.V.; CARVALHO, F.G. de. Matéria orgânica e propriedades físicas de um Argissolo Amarelo coeso sob sistemas de manejo de cana-de-açúcar. Revista Brasileira de Engenharia Agrícola e Ambiental, v.10, p.579585, 2006. DOI: 10.1590/S1415-43662006000300007.

SILVA, A.J.N.; RIBEIRO, M.R.; CARVALHO, F.G.; SILVA, V.N.; SILVA, L.E.S.F. Impact of sugarcane cultivation on soil carbon fractions, consistence limits and aggregate stability of a Yellow Latosol in Northeast Brazil. Soil and Tillage Research, v.94, p.420-424, 2007. DOI: 10.1016/j.still.2006.09.002.

SILVA, I.R. da ; MENDONÇA, E. de S. Matéria orgânica do solo. In: NOVAIS, R.F.; ALVAREZ V., V.H.; BARROS, N.F. de; FONTES, R.L.F.; CANTARUTTI, R.B.; NEVES, J.C.L. (Ed.).
Fertilidade do solo. Viçosa: Sociedade Brasileira de Ciência do Solo, 2007. p.275-374.

SISTI, C.P.J.; SANTOS, H.P. dos; KOHHANN, R.; ALVES, B.J.R.; URQUIAGA, S.; BODDEY, R.M. Change in carbon and nitrogen stocks in soil under 13 years of conventional or zero tillage in southern Brazil. Soil and Tillage Research, v.76, p.3958, 2004. DOI: 10.1016/j.still.2003.08.007.

SIX, J.; CONANT, R.T.; PAUL, E.A.; PAUSTIAN, K. Stabilization mechanisms of soil organic matter: implications for C-saturation of soils. Plant and Soil, v.241, p.155-176, 2002. DOI: 10.1023/A:1016125726789.

SOUZA, G.S. de; SOUZA, Z.M. de; SILVA, R.B. da; BARBOSA, R.S.; ARAÚJO, F.S. Effects of traffic control on the soil physical quality and the cultivation of sugarcane. Revista Brasileira de Ciência do Solo, v.38, p.135-146, 2014. DOI: 10.1590/S010006832014000100013.

TAN, Z.; LAL, R.; OWENS, L.; IZAURRALDE R.C. Distribution of light and heavy fractions of soil organic carbon as related to land use and tillage practice. Soil Tillage Research, v. 92 p.53-59, 2007. DOI: 10.1016/j.still.2006.01.003.

TORRES, J.L.R.; PEREIRA, M.G.; MORAES, A.G.L. de; BEUTLER, S.J. Frações granulométricas e oxidáveis da matéria orgânica em sistemas de colheita de cana-de-açúcar. Revista Caatinga, v.27, p.16-23, 2014.

Recebido em 26 de agosto de 2015 e aprovado em 28 de março de 2016 